\title{
INTERVENIR AUTREMENT DANS LES MILIEUX ÉDUCATIFS : REPENSER LES ASSISES ÉPISTÉMOLOGIQUES DES SCIENCES DU DÉVELOPPEMENT DE L'ENFANT
}

\author{
David Auclair \\ Ph. D. Chargé de cours en sociologie \\ Département de sociologie \\ Université du Québec à Montréal
}

\author{
auclair.david@uqam.ca
}

\section{Résumé}

Durant les années 1920-30, Lev. S. Vygotskij (1896-1934) s’intéresse aux médiations sémiotiques de la vie mentale afin d'étudier le développement des fonctions psychiques, émotionnelles et physiques de l'enfant. À cheval entre la pédologie et la défectologie, passionné d'art, de philosophie et de littérature, il sépare judicieusement l'âge mental de l'âge scolaire, mais aussi les savoirs cliniques des réalités éducatives. Toujours d'actualité, bien que demeuré en chantiers de son vivant, le projet éducatif vygotskien insiste sur le caractère dialectique du développement. Si le courant organiciste peut prétendre que le « petit d'homme apprend toujours mieux les faits qu'il découvre plutôt que les principes qu'on lui impose » (Piaget, 1997), il en va tout autrement pour celles et ceux qui préconisent l'approche historico-culturelle vygotskienne. Dans cet article, nous montrons que les interventions et les apprentissages varient historiquement en fonction des orientations épistémologiques, des contextes, mais aussi des méthodes préconisées pour régulariser et pour « performer» dans des cadres historiquement construits (signifiants). À l'école comme dans les familles, il s'avère impossible de se soustraire à l'obligation de privilégier les expériences appuyées sur des médiations culturelles, à la fois langagières et historiques. À l'instar de Vygotskij, nous pensons que les « acteurs significatifs » doivent privilégier le diagnostic pédagogique à l'aide d'interventions savamment orchestrées autour de la zone de développement proche (ZDP).

Mots clés : apprentissages, culture, développement, éducation, épistémologie

\begin{abstract}
During the 1920s-30s, Lev. S. Vygotskij (1896-1934) is interested by the semiotic mediations of mental life in order to study the development of the psychic, emotional and physical functions of the child. Straddling pedology and defectology, passionate about art, philosophy and literature, he judiciously separates mental age from school age, but also clinical knowledge from educational realities. Still topical, although still under construction during his lifetime, the vygotskian educational project insists on the dialectical character of development. If the organicist current can claim that the "little man always learns better the facts that he discovers rather than the principles that are imposed on him" (Piaget, 1997), it is quite different for those who advocate the vygotskian
\end{abstract}


cultural-historical approach. In this article, we show that interventions and learning vary historically according to epistemological orientations, contexts, but also the methods recommended to regularize and to "perform" in historically constructed frameworks (signifiers). At school and in families, it is impossible to escape the obligation to favor experiences based on cultural mediations, both linguistic and historical. Like Vygotskij, we believe that the "significant actors" must privilege the pedagogical diagnosis with the help of skillfully orchestrated interventions around the near development zone (ZDP).

Keywords: learning, culture, development, education, epistemology

\footnotetext{
Note d'auteur

Toute correspondance concernant cet article doit être adressée à David Auclair, Département de sociologie, Université du Québec à Montréal, local A-5055, 1255, St-Denis, Montréal (Québec) H2X 3R9, auclair.david@uqam.ca
} 
Revue internationale du CRIRES: innover dans la tradition de Vygotsky (2021) ISSN 2291-6717, vol 5, no 2, 3-18

Intervenir autrement dans les milieux éducatifs : repenser les assises épistémologiques des sciences du développement de l'enfant

\section{Introduction}

À la fin des années 1920 et au début des années 1930, Vygotskij a produit une théorie philosophique et scientifique du développement de l'enfant radicalement nouvelle sur des bases épistémologiques qui renvoient à la philosophie marxiste ${ }^{1}$. Il cherchait à mettre les sciences du développement de l'enfant sur les rails de l'interdisciplinarité afin de les sortir des apories des approches logico-formelles trop enclines à réduire et à fragmenter la vie mentale et affective ${ }^{2}$. D'après lui, le développement est « double ». D'abord, il apparait comme fonction du comportement social, c'est-à-dire comme catégorie interpsychique : une fois entré en contact avec les personnes signifiantes de son milieu, l'enfant s'approprie pour lui-même les signes et les instruments conventionnels (historiquement négociés). Les processus d'appropriation et d'intériorisation sont pour le développement ontogénétique « des conditions de fonctionnement et d'évaluation des actions humaines [...] qui fournissent la logique d'implication de significations qui se superpose au psychisme pratique et qui le réorganise » (Bronckart, 2002, p. 49). Ensuite, la capacité du sujet à s'approprier les signes et les instruments de sa culture passe invariablement par des médiations sociosymboliques et sémiotiques. Ces dernières provoquent chez le sujet une véritable révolution intérieure (intrapsychique). C'est ainsi que le sujet organise la vie sociale sous les pressions des milieux de vie en s'appropriant les artefacts culturels pour en faire des instruments psychologiques. C'est à cette condition qu'il apprend à penser par lui-même et que sa conscience se développe, car dans les mouvements multiples et contingents de ses expériences, il est constamment confronté au monde extérieur (Auclair, 2020).

En s'appropriant les signes culturels, l'enfant apprend aussi à connaitre. Une fois intériorisés, ces signes sont « comme des enveloppes fédérant des représentations individuelles » (Bronckart, 2002, p. 51). Ils sont aussi des instruments normatifs de coopérations, de communication et d'interventions. Pour l'illustrer, en commençant à penser, la conscience de l'enfant se développe comme " contact social avec soi-même » (Clot, 2003). Par exemple, avec l'apparition du langage intérieur, l'enfant peut se représenter ce qui est absent. Il « transforme » alors la réalité selon des règles conventionnelles qu'il adopte, mais il apprend aussi à coder ses expériences dans un «affrontement » avec le langage adulte (Dumont, 1981, p. 18). Plus qu'une action, le langage en est sa condition et la présuppose : " avant que je parle, une réserve de significations, de modèles et de règles est disponible dans le langage » (p. 17). Vous verrez plus loin que si l'action est centrale chez Piaget, c'est le langage qui prédomine chez Vygotskij.

Dans la première partie de cet article, nous proposons une critique du paradigme psychopédagogique organiciste. Notre réflexion est socioculturelle et historique. Nous considérons que l'éducation contribue à la dynamique de développement des fonctions psychiques, mais aussi à la configuration de la vie sociale : « [La situation sociale de développement] détermine totalement et complètement les formes et la voie par lesquelles l'enfant acquiert de nouveaux traits de sa personnalité [...] cette voie où le social devient individuel »(Vygotskij, 2017, p. 228). La

\footnotetext{
${ }^{1}$ Par la suite, nous écrivons Vygotskij, et non Vygotski ou Vygotsky, suivant la translittération internationale adoptée notamment par l'UNESCO.

2 Jean-Claude Quentel (2008) sépare judicieusement les développements effectifs des enfants (catégorie psychologique) et des jeunes (catégorie sociologique) de ceux des adultes. Très près en ce sens des intérêts scientifiques de Vygotskij, Quentel explique que les périodes et les fonctions psychiques et physiques des développements pendant la petite-enfance, puis pendant l'âge scolaire dépendent et rendent possibles des « néoformations » radicalement nouvelles pour la personne. Les changements développementaux à l'âge adulte sont d'une autre nature, car ils offrent plus de stabilité dans le temps et n'apparaissent plus comme de véritables révolutions psychologiques (mentales et affectives).
} 
deuxième partie approfondit certaines fonctions psychiques supérieures, mais aussi des catégories clés de l'épistémologie vygotskienne (conscience, mémoire, attention volontaire et imitation). Nous verrons comment ces dernières donnent naissance à la « personnalité consciente de l'enfant ». Autant sur le plan logique que sur le plan affectif, une dialectique de la contradiction est à l'œuvre dans la zone de développement proche (ZDP). En conclusion, nous insistons sur le fait que le développement humain est plus qu'une « accumulation graduelle et lente de changements isolés » (Sève, 2002, p. 253).

\section{Les paradigmes psychopédagogiques : entre approches organicistes et approches socioculturelles}

Chez Vygotskij, les médiations symboliques et sémiotiques traversent les âges et pénètrent les milieux de vie en produisant du sens et des significations. Le principal enjeu de l'éducation devrait être de faciliter les processus d'appropriation de ces médiations. Or, nous verrons dans les prochaines sections que le paradigme psychopédagogique oriente les pratiques éducatives bien différemment selon que l'on adhère aux approches dites organicistes ou aux approches dites socioculturelles. À vrai dire, ces deux approches ouvrent sur des applications radicalement opposées (en tension) :

Plus qu'un simple rapport extérieur entre deux disciplines, ce paradigme [psychopédagogique] instaure un découpage épistémologique qui implique en général pour la psychologie l'exclusion, de son champ de recherche, de l'intervention éducative et l'installation comme son objet du sujet individuel et de son comportement isolé [...]; et pour la pédagogie une perte importante de substance qui la fait glisser soit vers la spéculation philosophique ou morale soit vers l'application a-théorique. (Schneuwly, 1997, p. 27) ${ }^{3}$

Ce comportement isolé, dont parle Schneuwly, est « objet de contrôle » pour l'instrumentalisme psychopédagogique. Il devient une « performance » pouvant être comparée à d'autres comportements isolés. Or, il n'existe pas de vérités pratiques (empiriques) invariables en enseignement, comme il n'y a pas exclusivité des méthodes pédagogiques pour apprendre et transmettre (l'idéalisme psychopédagogique est une abstraction non scientifique). En prenant le temps de bien y réfléchir, s'en tenir aux performances scolaires pour évaluer la normalité développementale des élèves sur une base comportementaliste demeure un paradoxe (Bruner, 2015). Sous certaines acceptions scientifiques et techniques (psychopédagogiques) qui réduisent et déterminent l'intelligence, qui instrumentalisent les rapports socioaffectifs et la maturité infantile, se dévoile une structure logico-formelle de nature évolutionniste et positiviste (organiciste). Pour sortir de l'ornière « applicationniste » comprise comme technique séparée de la connaissance scientifique, Vygotskij élabore une épistémologie moniste et dialectique. Il propose également un projet éducatif (laissé en chantier) dans lequel développement,

\footnotetext{
3 Nous préférons parler de paradigmes psychopédagogiques, car d'une approche à l'autre, les pratiques pédagogiques se sont complexifiées depuis les années 2000 sous les influences de facteurs techniques et sociaux comme les outils technopédagogiques (outils TIC), les pédagogies adaptées, les pédagogies dites inclusives, les pratiques psychiatriques et psychopathologiques, mais aussi la nosographie de troubles comme l'anxiété ou le déficit de l'attention. Nous pensons néanmoins que cette apparente diversification des moyens et des techniques d'enseignement n'efface pas la tendance lourde qui consiste à expliquer le développement à partir de rapports normatifs biomédicaux et des seuils de performances (aussi nommés des marqueurs).
} 
apprentissage et enseignement forment un tout indivisible. Quelques remarques permettront de comprendre sa méthode et son épistémologie.

\section{L’épistémologie de Vygotskij comme projet éducatif}

Tout d'abord, Vygotskij constate que les approches logico-formelles en éducation réduisent les fonctions ainsi que les rôles joués par les adultes sur le développement ontogénétique (individuel). Par la suite, il affirme que les moyens pédagogiques sont comme la technique dans les sciences de la physique : « la pédagogie se rapporte à la pédologie comme la technique à la physique. La physique élabore des lois de la nature en tant que telles, alors que la technique utilise ces lois. C'est exactement la même chose pour la psychologie et la pédologie qui établissent des lois du développement de l'enfant, alors que la pédagogie construit l'enseignement de l'enfant sur la base de ces lois. » (Vygotskij, 2018, p. 380) C'est le cœur de son épistémologie pédologique, mais aussi de ses critiques envers la psychologie génétique piagétienne et le formalisme logique.

La pédagogie chez Vygotskij possède une puissance inédite et transformatrice pour au moins deux raisons. Premièrement, en devenant les porteurs de projets scolaires réfléchis, le corps enseignant admet une historicité et une responsabilité qui ont pour finalité de le rendre plus autonome face aux doctrines réductionnistes et autres exigences méthodologiques logicoformelles. Réduire l'intelligence et les rapports socioaffectifs à des performances mesurables empêche de saisir pleinement les mouvements ainsi que la complexité des périodes pendant lesquels l'enfant s'approprie et intériorise les éléments de sa culture : "Les données normatives ne doivent pas être utilisées mécaniquement ou de manière purement psychométrique. Nous ne devons pas uniquement mesurer l'enfant, nous devons l'interpréter » (Vygotskij, 2018, p. 242). En récusant la fracture qui sépare les sciences et les pratiques des instruments psychologiques et culturels, Vygotskij sert entièrement le projet éducatif. Ce qu'il comprend comme un « diagnostic de développement » prend racine dans la pédologie et propose de faciliter l'intégration sociale des élèves, mais aussi les rôles des acteurs significatifs, en adoptant une position non déterministe de ce que devrait être le devenir ontogénétique. Autrement dit, en voyant l'enfant comme un être en devenir au lieu de le traiter comme un objet de l'instrumentalisation formaliste et organiciste (évolutionniste), le corps enseignant conscient de ses pratiques contribue à une véritable émancipation des sujets historiques, en théorie, mais aussi en pratique ${ }^{4}$. Deuxièmement, ce changement radical des mentalités permet à Vygotskij de montrer comment les courants épistémologiques, mais aussi le choix des méthodes éducatives, conditionnent les pratiques scolaires et les activités d'enseignement au même titre que le développement des individus et des sociétés. Sur le plan de l'éthique scolaire, on gagnerait selon lui à sortir l'école des apories technoscientifiques et du réductionnisme biologique ${ }^{5}$. De haut en bas, de bas en haut, rien ne sépare

\footnotetext{
${ }^{4}$ Vygotskij propose une méthode clinique indirecte de nature génétique et comparative qui se construit dans les activités et dans les milieux de vie. Sur ce point, le problème clinique qu'il dénonce tient dans les conséquences sociales ainsi que dans les réductionnismes formalistes qui empêchent de comprendre le développement intégral de l'enfant. Il donne un exemple de cet empêchement dans le texte Le problème de l'âge : "Si l'on amène un enfant en consultation de pédologie en disant qu'il a des difficultés de développement mental, qu'il a des difficultés de compréhension, qu'il a du mal à mémoriser et que le psychologue après examen pose le diagnostic : coefficient de développement bas-retard mental, là non plus, il ne peut rien expliquer, ni pronostiquer, ni aider sur le plan pratique » (Vygotskij, 2018, p. 243).

${ }^{5}$ Les technosciences s'appuient sur la science, mais dans un rapport technique, c'est-à-dire comme une performance qui sert une finalité attendue. Si l'esprit de la science implique le doute, l'esprit des technosciences cherche plutôt à agir pour faire réagir, pour provoquer une transformation sur laquelle elle pourra agir à nouveau à l'aide de procédés brevetés et rentables.
} 
réellement les pratiques de la théorie. La réussite relative de l'école traditionnelle ne tenait-elle pas à sa capacité à refermer le milieu scolaire sur lui-même en proposant une « culture seconde », c'est-à-dire « relativement coupé de son milieu habituel d'existence [ce qui a permis] à l'enfant [de se] rapporter à l'univers de l'écrit [et de la lecture] comme à un référent particulier » (Dumont, 1981, p. 26)?

\section{Quelques fondements généraux des sciences du développement de l'enfant : la perspective socioculturelle}

Dans les approches socioculturelles, le langage est à la fois médiation et instrument psychologique. Il est plus qu'une conduite et plus qu'une action, car il en organise et en légitime le sens et la portée. C'est ce qui fait dire à Vygotskij que l'acteur significatif intervient dans la ZDP pour aider l'enfant à organiser ses expériences, mais aussi pour mettre en place des pratiques signifiantes qui répondent aux aspects essentiels du développement humain (intellectuel, émotionnel, affectif, relationnel). C'est plus un état d'esprit qu'une règle à suivre scrupuleusement, étant donné que les formes et les fonctions développementales sont multiples et variables selon la nature des rapports sociaux. Précision : il est préférable de parler d'une zone de développement proche plutôt que d'une zone proximale de développement, parce que « ce n'est pas la zone qui est proximale, mais le développement » (Leopoldoff Martin \& Schneuwly, 2018, p. 235).

Ensuite, au plus près des aptitudes et des compétences de l'enfant, l'adulte significatif lui apprend à parler pour s'exprimer et pour assumer ses conduites. Ceci passe in vivo par des conventions sociales négociées historiquement. C'est le propre des approches socioculturelles d'admettre que l'enfant n'est pas autonome à la base, mais qu'il le devient par ces dialogues et ces « affrontements » à partir desquels il cherche à offrir à l'adulte un « contre-rendu » de ses activités et de ses apprentissages. Par exemple, en analysant le soliloque chez l'enfant, qui est à la fois expression et capacité de résolution de problèmes avant d'être un langage intérieur, Vygotskij comprend le langage comme un horizon doué d'une historicité.

Langage social - langage égocentrique - langage intérieur, tel est donc le schéma dans son ensemble. Il s'oppose, d'une part, à celui de la théorie traditionnelle sur la formation du langage intérieur : langage extériorisé - chuchotement — langage intérieur, et, d'autre part, au schéma de Piaget, où les principaux stades génétiques dans le développement de la pensée logique verbale s'ordonnent ainsi : pensée autistique non verbale — langage égocentrique et pensée égocentrique - langage socialisé et pensée logique (Vygotskij, 1997, p. 116).

L'approche historico-culturelle que propose Vygotskij saisit dialectiquement que les sciences du développement de l'enfant ne sont pas figées dans le temps ; que celles-ci doivent répondre et être confrontées au réel effectif, historique, pour servir la cause de l'éducation, et vice-versa: « L'adulte, on l'aura compris, ce n'est pas seulement la mère, le père, mais tous ceux qui sont les titulaires d'un héritage de techniques, d'informations et de sens » (Dumont, 1981, p. 18).

Selon la perspective historico-culturelle, la reconstruction artificielle de la nature développementale autour des concepts d'autonomie, d'adaptation, de coopération spontanée entre enfants du même groupe d'âge, pour ne nommer que ces catégories piagétiennes, n'explique pas le développement réel, mais en produit une image qui tient dans les observations et les conclusions cliniques ou expérimentales (Vygotskij, 1983/1994) : «Et ce goût des lignes droites, ce caractère 
linéaire dans la délimitation des sciences qui nous vient en héritage de la logique formelle, se retrouve impuissant face à la complexité et à l'imbrication réelle des phénomènes étudiés par chacune de ces sciences » (Vygotskij, 2018, pp. 374-375).

\section{Quelques fondements généraux des sciences du développement de l'enfant : la perspective organiciste}

Il va sans dire que Piaget a contribué significativement aux avancées des sciences du développement humain. Il en a donné les premières acceptions scientifiques et expérimentales sérieuses en montrant que la première intelligence de l'enfant est organisée autour d'un schématisme sensori-moteur. Les formes représentatives à ce stade demeurent toutefois dépendantes des objets; c'est ce qu'il nomme l'intelligence pratique. Pour le dire autrement, la morale et la conscience sont alors adaptées au psychisme. Ces constatations expérimentales lui permettent de critiquer avec justesse une éducation trop rigide dans laquelle les règles et les normes de l'adulte ne peuvent pas être assimilées par les jeunes enfants. C'est seulement à partir du double jeu des mécanismes récursifs d'assimilation et d'accommodation qu'une intériorisation d'unités de représentation autonomes peut faire place à la pensée (abstraite). Or, Piaget, à la différence de Vygotskij, accorde très peu d'importance aux signes langagiers et aux transmissions sociales agissants sur les fonctions psychiques et le développement affectif : «Les opérations de la pensée [...] tiennent aux coordinations générales de l'action [...] et non pas au langage et aux transmissions sociales particulières, ces coordinations générales de l'action se fondant ellesmêmes sur les coordinations nerveuses et organiques qui ne dépendant pas de la société » (cité dans Bronckart, 2002, p. 32). En s'appuyant sur les thèses évolutionnistes de Herbert Spencer, la perspective organiciste piagétienne comprend les opérations intellectuelles comme des mécanismes naturels qui passent du simple au complexe (Blais et al., 2010). Ses critiques à l'endroit de l'école durkheimienne sont évocatrices de cette prise de position. Selon lui, l'autorité adulte doit se subordonner au self-governement, c'est-à-dire à l'engagement mutuel et à la coopération volontaire (Piaget, 1997).

C'est ainsi que J. Piaget peut être considéré comme le représentant par excellence d'une impossible réconciliation : celle d'une pédagogie à vocation collective, qui hérite en même temps d'une tradition qui allie la psychologie de l'enfant à une vision de l'éducation évolutionniste et progressiste, individualiste et libertaire. Il n'est ni « responsable » ni seul représentant de cette philosophie implicite, il en donne plutôt une expression achevée. (Ottavi, 2009, p. 288)

L'idée générale qui se dégage de cette philosophie scientifique consiste à accompagner l'enfant en le laissant découvrir et reconstruire le monde par lui-même. L'engagement adulte se doit d'être minimaliste, car toutes les interventions «verticales » risquent d'interférer et de modifier les conduites en contraignant unilatéralement l'initiative privée (Spencer, 1866). Du côté de l'organicisme, les interventions scolaires doivent toujours être indirectes, car l'enfant apprend mieux les faits qu'il découvre par lui-même plutôt que les principes qu'on lui impose. Les intervenants deviennent des observateurs, qui suivent de près les actions individuelles sans les orienter ou les rendre obligatoires. 


\section{De l'approche organiciste à l'approche socioculturelle : deux conceptions divergentes de l'autonomie}

Plusieurs intervenants analysent ce double déterminisme, organiciste et logico-formel, avec un brin de suspicion (Quentel, 2008). D'un côté, il est dit que l'enfant est autonome par nature, mais de l'autre, dès qu'il y a déficit perçu, débordement ou écart possible, prévisible, on réclame des interventions formelles, mesurables, souvent de nature comportementaliste. Il est possible de remarquer aussi que la production de ces nouveaux savoirs « descriptifs » conduit à un réductionnisme dangereux qui légitime le recours systématique à la médicalisation des conduites et des humeurs. Or, Vygotskij a déjà signalé les limites «thérapeutiques » d'une telle gestion de la nature humaine dans son traité de défectologie. Cela ne date pas d'hier que les performances scolaires ou émotionnelles des élèves sont scrutées à la loupe, hors contexte.

En suivant l'approche socioculturelle vygotskienne, au lieu de se pencher sur les seuls troubles, vulnérabilités et autres pathologies de la vie quotidienne, il est entendu que l'enfant a sa propre histoire (Vygotskij, 1983/1994) ${ }^{6}$. Il ne suffit pas de prendre une photographie des aptitudes actuelles pour les isoler, les mesurer et les comparer. Vygotskij le dit clairement : le diagnostic pédagogique du développement oblige à suivre et à comprendre les acquis à la fin d'une période, pas au début du processus éducatif. Le fait de privilégier le diagnostic pédagogique afin de reconnaitre les aptitudes potentielles (encore non réalisées) de chaque personne implique aussi que l'autonomie n'est pas un fait de nature, mais un fait de culture. On devient autonome, c'est-à-dire capable de s'approprier les éléments de sa culture pour soi-même, parce que l'on imite et intériorise les façons de faire et de dire, les manières d'être au monde, d'être dans le monde. Le développement est toujours plus qu'une somme de comportements et de symptômes observés.

Néanmoins, il va sans dire qu'il n'est pas facile de tracer une ligne de partage entre les bénéfices et les dangers qui s'enchainent et se mélangent dans le contrôle comportementaliste en contexte scolaire :

Les problèmes pénibles de la discipline et de l'indiscipline dans la société de masse rendent l'extension de telles méthodes de contrôle à des catégories non médicales extrêmement tentante pour la gestion sociale. [...] Allons-nous induire des dispositions à l'apprentissage chez les écoliers par l'administration massive de drogues, contournant l'appel à la motivation autonome? (Jonas, 2013, p. 41)

Rappelons que les contingences historiques ont de tout temps obligé les humains à intérioriser d'autres conditions, d'autres nécessités, d'autres obligations qui se sont imposées par la force des choses, et que ceci n'est jamais apparu sans certains mécanismes de régulation. Les manières de réguler les dynamiques sociales sont forcément multiples et variables. Les chemins menant à l'autonomie répondent aux mêmes exigences éthiques et contingentes. Reste que les valeurs et les principes de responsabilités sous-jacents ne sont pas les mêmes et que « l'enseignant apprend à l'enfant non pas ce qu'il peut déjà faire de manière autonome, mais ce qu'il ne sait pas encore faire seul et peut l'accomplir grâce à l'enseignement » (Vygotskij, 2018, p. 241). À ce propos, Vygotskij explique que le sujet « est plein à chaque minute de possibilités non réalisées »(Clot, 2003, p. 22).

\footnotetext{
${ }^{6}$ Nous parlons d'enfants si nous référons à l'historicité et d'élèves si nous référons à l'agentivité. À l'école, suivant le raisonnement de Vygotskij, il vaut mieux parler d'élèves pour référer à cette agentivité sans jamais perdre de vue qu'ils sont des porteurs d'historicité.
} 
Les périodes de développement dont parle Vygotskij sont toujours fluctuantes. Elles sont à la fois négatives et positives, ce qui impose d'agir « pédagogiquement » sur les conduites de l'enfant non pas à partir de son agentivité (l'enfant compris dans sa synchronicité et ses résultats — c'est-à-dire de ses performances), mais bien à partir de sa personnalité (l'enfant compris dans son historicité propre). Dans la même optique, Bronckart critique lui aussi avec justesse les dispositions logico-formelles qui séparent les apprentissages et le développement, mais aussi les sciences psychologiques et les moyens pédagogiques :

Ce statut de la pédagogie comme « champ d'application » de disciplines fondamentales constitue un paradoxe, qui est particulièrement clair dans le domaine de la psychologie cognitive : les expériences sont réalisées (la plupart du temps) en situation scolaire, sans que soient pris en considération les aspects socio-institutionnels de cette même situation; les données recueillies sont ensuite analysées dans les cadres d'une psychologie universalisante et a-sociale, et elles sont enfin réinjectées dans le milieu scolaire, au titre de données fondamentales (d'éléments de « compétences ») évidemment soumises aux aléas de tout fonctionnement effectif, c'est-à-dire de toute « performance » (Bronckart, 1985, p. 19).

Ne rencontrons-nous pas à nouveau, dans les contextes comportementalistes et hygiénistes actuels, les mêmes problèmes éducatifs entre ce qui est désiré (comprendre le développement intégral de l'enfant en prenant en compte son milieu de vie et son histoire) et ce qui est obtenu dans le réel effectif (une mise à l'écart et une séparation des aptitudes qui répondent à des critères objectivables de compétences)?

Il faut maintenant réfléchir sur la portée éducative, politique et scolaire, des approches socioculturelles. Tout d'abord, ces approches accordent toute leur importance aux interactions entre les adultes et les enfants. Dans un sens anthropologique qui refuse de niveler les rapports éducatifs dans un égalitarisme politique, l'enfant n'entre pas dans la catégorie des opprimés qu'il faut libérer de la tutelle adulte (Quentel, 2008) ${ }^{7}$. Si Piaget concevait l'enfant comme structurellement différent et fonctionnellement identique de l'adulte, Vygotskij le conçoit comme structurellement et fonctionnellement différent. Il ne s'agit donc pas de comprendre l'enfant générique, mais l'enfant en tant qu'élève (Schneuwly, 1997, p. 43) : "Considéré de ce point de vue, l'apprentissage ne coïncide pas avec le développement, mais active le développement mental de l'enfant, en réveillant les processus évolutifs qui ne pourraient être actualisés sans lui » (Vygotskij, 1985, p. 112). Ces moments privilégiés de l'apprentissage scolaire s'avèrent constitutifs d'une autre réalité individuelle et sociale, car ces traits et autres fonctions psychiques supérieures se développent de manière contingente, concomitante, historique, et sont non arbitraires.

Même si elles sont par essence contraignantes, les pratiques d'enseignement dirigées permettent de prendre conscience à chaque instant des responsabilités et des devoirs qui incombent aux adultes de faire advenir les enfants dans un monde déjà existant. L'autonomie est une quête

\footnotetext{
${ }^{7}$ Nous considérons l'égalitarisme politique et éducatif en Occident comme une posture idéologique qui ne reconnait plus la légitimité des hiérarchies sociales. Si les formes réelles du pouvoir dans les sociétés traditionnelles occidentales demeuraient verticales, le paradigme éthique actuel en éducation ou en politique tourne autour d'une horizontalité et d'une transparence. Le " système scolaire " serait lui aussi autoréférentiel et nous ne devrions agir que sur les réactions et les conduites (par différents mécanismes de reddition). Avec Vygotskij, nous pensons que la transmission et les interactions en éducation imposent toujours une verticalité des rapports entre les adultes et les enfants.
} 
qui vient avec des responsabilités. Vygotskij répète à bon escient que le seul bon enseignement est celui qui précède le développement.

\section{L’importance de la pensée dialectique en éducation}

Nous avons vu dans la première partie que la perspective logico-formelle traite l'enfant générique comme un « objet » absolu, souvent sans prendre en compte les a priori normatifs et culturels qui agissent hors des murs du Yalta scientifique et des différentes expérimentations cliniques qu'elle préconise (Clot, 2012, pp. 143-144). A contrario, nous avons montré que l'approche socioculturelle s'intéresse à l'élève comme personne ayant sa propre histoire. Le programme épistémologique vygotskien étudie le développement de l'enfant sur un axe non linéaire. En proposant un autre cadre pratique et théorique qui associe la philosophie, la littérature, les sciences psychologiques et sociologiques, Vygotskij critique les limites des tests psychométriques, mais aussi l'idéalisme positiviste qui détermine des seuils d'intelligence et de maturité à partir d'un âge chronologique donné et d'indicateurs psychométriques. Il dénonce le manque de souplesse des expérimentateurs face aux exigences sociales, soit à ce que l'on entend par performances et à ce que l'on conçoit comme la réussite. Vygotskij s'attaque à ces perspectives ainsi qu'à leur méthodologie propre en s'appuyant dans les sciences de la psychologie sur les principes de la dialectique de la contradiction développée par Marx :

La première, le préformisme, aboutit à une vision du développement comme accroissement de ce qui est déjà là ; la seconde repose sur une conception du développement comme déterminé par le milieu et l'enfant comme être passif ne réagissant pour l'essentiel qu'aux stimuli empruntés au milieu. [...]. Elles constituent de fait la négation du développement, qui apparaît comme processus stérile dès lors que tout est déjà en germe ou dans le milieu. (Leopoldoff Martin \& Schneuwly, 2018, p. 33.)

Pour Vygotskij, bien que le développement réel de l'enfant suive des lignes principales et des lignes secondaires, il n'y a pas concrétisation, équilibration, puis dépassement d'un stade vers un autre. Il accorde aussi beaucoup d'importance à l'imitation comme « facilitateur » du développement (apprendre en imitant, refaire les problèmes à l'aide d'exemples offerts et imposés). Cela permet de considérer que la maturité est « temporalité multiple» (p. 235), car elle ne peut pas être considérée seule sans passer par la direction pédagogique. Il insiste sur le fait que la maturité et l'autonomie doivent être liées à la ZDP comme résultats ou comme le fruit « du développement de l'âge » : «Le changement dans la conscience de l'enfant émerge sous une forme définie de son existence sociale particulière à un âge donné. Voilà pourquoi la maturation d'une néoformation se rapporte non pas au début, mais à la fin d'un âge donné » (Vygotskij, 2018, p. 228). À la base de sa théorie pédologique et de ses analyses en défectologie, nous retrouvons les catégories épistémologiques suivantes : âge de développement et périodisation des fonctions et des mécanismes, élaboration et appropriation du sens à partir de significations culturelles et langagières, âge de développement et écart entre le niveau intellectuel réel et potentiel. C'est ce qui nous amène à nous intéresser aux fonctions supérieures. 


\section{Le langage et la culture comme médiations : conscience, attention volontaire, mémoire et imitation au service du développement}

Les médiations symboliques et sémiotiques sont essentielles à la vie mentale (psychologique) du sujet ${ }^{8}$. Nous l'avons mentionné, à l'aide d'instruments psychiques et d'outils techniques, l'individu s'approprie les artefacts culturels de la société historique (outils, parole, écriture, calcul, etc.). Bruner écrit à ce propos que la culture est une espèce de boîte à outils et que l'éducation familiale et scolaire doit permettre à l'enfant de s'approprier le sens et les éléments les plus significatifs de celle-ci. Il précise que les significations s'inscrivent dans des systèmes symboliques « enracinés dans la culture et le langage » (Bruner, 2015). Par essence, d'une implacable constance, " la pensée et le langage sont la clef pour comprendre la nature de la conscience humaine »(Vygotskij, 1997, p. 509). En reprenant la formule de Clot (2003), « [1]a conscience est le passager clandestin de la psychologie expérimentale » (p. 10). Elle permet de consolider les expériences, car « l'activité psychologique en conflit du sujet » relie le physiologique et le psychique dans les apprentissages (appropriation et intériorisation) ${ }^{9}$. La conscience est mémoire du geste, de l'émotion, du mot et de l'intention, car elle s'instruit dialectiquement des médiations en les retournant sur elles-mêmes pour en faire les outils privilégiés des expériences scolaires. La théorie psychologique des affects de Vygotskij rencontre ici sa théorie des significations (signes et symboles).

De la sorte, si les médiations symboliques et sémiotiques sont si importantes dans l'approche historico-culturelle, c'est qu'elles traversent et structurent les fonctions psychiques et langagières, et ce, tout au long de la vie. Elles s'intercalent entre les aperceptions individuelles et celles du monde adulte, puisque l'enfant « commence à comprendre le langage avant de commencer à parler » (Vygotskij, 1983/1994, p. 134). Les médiations symboliques et langagières font que le développement humain est ce qu'il est bien au-delà de ses aspects sensori-moteurs et de ses réactions visibles (symptômes). Comme l'écrit Clot dans son introduction à l'ouvrage Conscience, inconscient, émotions (2003) :

Par sa médiation, le sujet « représente » son activité qu'il modifie du même coup. La conscience, dans l'expérimentation vygotskienne, n'est pas en deçà ou au-delà de l'expérience comme un État dans l'État. La conscience est une forme particulière de transformation de l'expérience ou d'échec de cette transformation (p. 15).

Conscience, mémoire et attention volontaire se trouvent donc liées dans les milieux éducatifs alors que les mécanismes de l'imitation produisent leurs effets constructifs.

\section{Esquisse vygotskienne sur l'attention volontaire}

Comme le disait déjà Vygotskij au début des années 1930 en parlant de l'attention volontaire, «le travail de distinction entre le fond et la forme, c'est-à-dire, la fonction d'attention volontaire avec la capacité d'orienter l'attention sur des facteurs de perception qui sont structurés entre eux afin d'attirer l'attention sur eux, cette fonction se développe tardivement » (Vygotskij, 2018, p. 334). Concrètement, l'éducateur doit stimuler et réguler les conduites, l'intérêt, la curiosité, tout en

\footnotetext{
${ }^{8}$ Le psychologique chez Vygotskij forme une catégorie plus générale qui intègre les fonctions psychiques et affectives.

${ }^{9}$ L'expression entre guillemets est de Y. Clot (2002, p. 23). La conscience vient avec les affects chez Vygotskij. C'est ainsi qu'il entend dépasser le parallélisme freudien qui « juxtapose » psychisme et conscience.
} 
insistant sur des projets qui « collent » aux capacités actuelles et potentielles des enfants. Conscient de sa pratique, il sait que l'apprentissage précède le développement, mais il sait aussi qu'un ordre doit être respecté. D'un point de vue plus fonctionnel, l'éducateur doit affiner la part volontaire de l'attention chez l'enfant, ce qui nécessite de réduire au maximum les distractions, mais, surtout, la dispersion. Or, cette part d'attention volontaire est une fonction psychique parmi tant d'autres et elle n'apparait pas avant un âge plus avancé (à partir de l'âge de 7 ans). Vygotskij se souvient assurément du projet pédagogique hégélien (Hegel, 1990) : la part volontaire de l'attention est une fonction qui se développe graduellement chez l'enfant, inégalement, alors que ce dernier demeure très sensible aux stimulations du milieu. C'est de cette façon que le côté volontaire de l'attention s'insère comme fonction supérieure dans ce que Vygotskij nomme un phénomène de second degré. Équipé de cette capacité mentale en mouvement, l'élève en situation scolaire rencontre et se trouve de nouveau confronté à son propre travail intrapsychique (c'est-à-dire à l'effort personnel). L'attention volontaire se développe principalement dans divers contextes culturels d'apprentissages. Ce qui est valable pour l'attention volontaire est également valable pour la mémoire. En effet, ces deux fonctions psychiques apparaissent inégalement dans le cours du développement sous l'effet des influences des milieux de vie. Ce ne sont donc pas des performances que l'on peut aisément mesurer. La mémoire est le terreau fertile de la pensée. Ces constats obligent à nouveau de devoir différencier les habitudes et les aptitudes dans le perfectionnement de l'intellect, mais aussi le défaut du handicap. Avec beaucoup de variabilité dans les capacités, il faut toujours se demander comment éduquer un enfant en s'intéressant, non pas à sa « maladie », mais à l'enfant lui-même.

\section{L'imitation et l'altérité au service de l'apprentissage}

En continuité avec ce qui précède, dans un texte intitulé « La collectivité comme facteur de développement de l'enfant handicapé », Vygotskij écrit que

[t]andis que le manque de développement des fonctions élémentaires se manifeste souvent en tant que conséquence directe d'un déficit quelconque (par exemple le retard dans le développement moteur chez l'enfant aveugle, le manque de développement du langage chez l'enfant sourd, la pensée insuffisamment développée chez l'arriéré mental, etc.), le manque de développement des fonctions supérieures chez l'enfant handicapé est souvent un phénomène ajouté, de second degré, qui représente une superstructure secondaire par rapport aux caractéristiques primaires (Vygotskij, 1983/1994, p. 169).

Le point est essentiel : pour intervenir et pour agir sur la conscience et les apprentissages, en éducation, mais aussi dans les pratiques d'enseignement, il est essentiel de saisir le travail du négatif comme formateur pour les individus (Sève, 1997 ; 2002). Nous pouvons même affirmer que « la négation de la vie est par essence contenue dans la vie elle-même » (Clot, 2003, p. 23).

Toute La Défectologie est habitée par le souci d'éviter concrètement la métamorphose de la déficience en handicap, que Vygotskij distingue soigneusement. [...] Il s'agit, ni plus ni moins, de contrer les risques de voir les enfants déficients se couper des forces vives de l'altérité en se soustrayant eux-mêmes aux sollicitations relationnelles du monde social. (Clot, 2003, pp. 37-38)

Selon Leopoldoff et Schneuwly (2018), trois facteurs contingents unissent les enseignements et le développement : « le fait incontestable que l'enseignement agit simultanément en retour sur le 
développement ; l'existence simultanée de deux niveaux de développement, l'actuel et le potentiel, ou proche; la différence fondamentale entre apprentissage scolaire et non scolaire » (p. 23). L'interaction de ces trois facteurs contingents a été largement appuyée par les réflexions vygotskiennes sur le langage écrit et la grammaire, plus particulièrement avec le chapitre 6 sur l'écriture dans Pensée et Langage. Une synthèse peut être envisagée : les adultes significatifs font plus qu'accompagner les enfants, ils contribuent à faire naître ce qui n'existe pas encore à l'état de nature.

Il reste un élément à couvrir. En éducation, contrairement à ce qui est largement valorisé dans les systèmes éducatifs actuels, Vygotskij voit l'imitation comme un instrument facilitateur du développement à l'intérieur de la ZDP. Dans le domaine intellectuel comme dans ses rapports affectifs, grâce à l'imitation, l'enfant que l'on guide (que l'on dirige) apprend toujours plus que s'il avait été laissé à lui-même :

En parlant ici d'imitation, nous entendons une imitation qui n'est pas mécanique, automatique, insensée, mais une action sensée, basée sur la réalisation imitée d'une quelconque opération intellectuelle. [...] Tout ce que l'enfant ne peut pas faire de manière autonome, mais qu'il peut apprendre ou accomplir en étant guidé ou en collaboration, à l'aide de questions subjectives ou une aide sur un point difficile, tout cela se rapportera pour nous au domaine de l'imitation. (Vygotskij, 2018, p. 237)

Nous disions plus haut avec Bronckart que, pour Piaget, le langage n'engendre pas le développement cognitif; il sert ce développement, mais toute nouvelle opération « se construit à partir de l'action du sujet » (Bronckart, 1977, p. 62.). La parole est traitée chez Piaget comme une action parmi tant d'autres. On retrouve aussi dans cette conception du développement naturel de l'intelligence le trait lamarckien de la transformation des caractères acquis (Ducret, 1984), car « [Piaget] n'a guère attaché d'importance à la nature même des objets et des événements que l'enfant devait traiter; l'importance du milieu est toujours mentionnée, mais elle n'est jamais véritablement prise en considération » (Bronckart, 1977, p. 63). Nous comprenons bien que c'est tout le contraire qui se produit dans la théorie socioculturelle vygotskienne.

Somme toute, il est toujours tentant d'associer la théorie constructiviste et génétique de Piaget à celle de Vygotskij, soit en injectant des éléments culturels dans la théorie du premier, ou bien en évoquant par-ci, par-là, les influences des milieux et du langage sur le développement infantile. Or, avec La Défectologie vygotskienne : les conséquences sociales et scolaires sont devenues trop importantes (médicalisation précoce, opérationnalisation biomédicale des activités scolaires, discriminations dites abusivement positives) pour maintenir un consensus entre deux approches qui sont diamétralement opposées sur le terrain des pratiques scolaires. Même si de nombreux éléments théoriques les rapprochent (Vygotskij, 1997), leurs assises méthodologiques et épistémologiques sont trop éloignées les unes les autres pour tenter un bricolage sans produire des amalgames fâcheux.

\section{Le diagnostic pédagogique et la ZDP comme nouveaux cadres éducatifs}

Nous avons insisté tout au long de ce travail sur le fait que le développement ontogénétique et les enseignements, mais aussi les interventions pédagogiques forment un tout indivisible :

Le développement de l'enfant ne suit pas comme une ombre l'activité didactique développée à l'école. C'est pour cela que les tests sur les résultats obtenus à l'école par l'enfant ne reflètent jamais le cours effectif de son développement. De fait, des rapports dynamiques 
d'interdépendance s'instaurent entre développement et apprentissage, rapports qu'on ne peut exprimer en une formule théorique artificielle, aprioriste, donnée une fois pour toutes (Vygoskij, 1985, p. 116).

La réalité comportementaliste des sciences logico-formelles agit sur le développement comme une analyse de second degré, elle est surajoutée, et conduit à un certain «fatalisme » éducatif. Avec Vygotskij, nous pensons que la vie scolaire peut transformer la nature de l'enfant. Le paradigme formaliste et explicatif se confirme comme « une doctrine de l'immobilisme, de l'innéité et de l'isolement total des phénomènes psychiques hors de toute réalité matérielle » (Vygotskij, 2018, p. 363). Il s'agit d'arguer contre ce « fatalisme » organiciste que tout « apprentissage humain est à la fois artificiel et naturel, et toujours en partie dépendant d'une des institutions humaines » (Bronckart, 1985, p. 19).

Avec la ZDP, l'écolier intériorise dialectiquement les tensions et les contradictions de sa culture. Ces contraintes extérieures obligent les sujets à se mouvoir, à s'émanciper et à étendre leurs capacités dans un devenir incertain devenu horizon indéfini rempli de possibilités non achevées. Cela se fait toujours à l'aide de processus d'enseignement correctement construits avec l'Autre (c'est le sentiment d'altérité). Apprendre à lire et à écrire requiert plus que des habitudes cristallisées, comme, par exemple, appliquer des règles orthographiques. La ZDP est une méthode dynamique non formaliste.

Concluons cette section avec une autre synthèse. L'idée est de tracer les contours d'un nouvel impératif proposant de (re)penser l'éducation en insistant sur les médiations sémiotiques et sociosymboliques du diagnostic pédagogique :

Évaluer le développement réel de l'enfant, c'est identifier et diagnostiquer « les processus encore non matures », c'est-à-dire tout ce que l'enfant ne peut faire encore de manière autonome, physiologiquement, intellectuellement, émotionnellement, ceci de la naissance à l'adolescence. L'apparition d'une zone de développement est liée aux moments de ruptures, à chaque âge. La modification du rapport entre une fonction donnée et le milieu se répercute sur l'ensemble du système des fonctions psychiques en le transformant. À chaque pas, pour peu qu'on l'accompagne, l'enfant se forge des instruments psychologiques nécessaires pour la suite de son développement (Leopoldoff Martin \& Schneuwly, 2018, p. 27).

La photographie des aptitudes et des compétences évoque un certain « état de réalisation » posé en amont du travail d'accompagnement. Nécessairement psychométrique, cette approche demeure insuffisante pour comprendre les mouvements dialectiques à l'œuvre entre l'éducation, le développement ontogénétique et les apprentissages. Les enfants entrent à l'école avec un bagage langagier et culturel déjà discriminant. En généralisant le propos, tous les intervenants en contexte scolaire visent l'autonomie de l'élève, mais, dans les faits, les moyens qui permettent de l'atteindre diffèrent radicalement selon que l'on attache cette autonomie à la nature biologique ou aux acquisitions sociales, culturelles et scolaires.

\section{Conclusion}

Autour de la périodisation de l'âge scolaire, Vygotskij présente en 1934, devant ses étudiantes et étudiants, un exposé que l'on peut lire en français sous le titre Le problème de l'âge (Vygotskij, 2018). Il parle dans ce texte des difficultés rencontrées dans les milieux scolaires à 
partir du moment où « [1] es formes externes des phénomènes étaient prises pour la nature des faits eux-mêmes » (Vygotskij, 2018, p. 205). Il renvoie à Marx qui explique que « [t]oute science serait superflue si l'apparence et l'essence des choses se confondaient » (p. 205). Il y a problème au moment où les rythmes scolaires imposés n'admettent plus la grande variabilité des apprentissages et des conduites individuelles :

Chaque âge possède une structure spécifique, unique et non duplicable. [...] Si nous nous arrêtons sur la conscience de l'enfant, entendue comme son « rapport au milieu » (Marx) et si nous considérons la conscience générée par les changements physiques et sociaux de l'individu comme l'expression intégrale des caractéristiques supérieures les plus essentielles dans la structure de la personnalité, nous verrons qu'au passage d'un âge à l'autre ce ne sont pas tant les aspects particuliers, partiels de la conscience qui croissent et se développent, ses fonctions ou ses moyens d'action particuliers; ce qui change en premier lieu, c'est la structure générale de la conscience qui à chaque âge se caractérise d'abord par un système de rapports et de dépendances précis existant entre ses différents aspects en particulier, les différentes facettes de son activité (p. 226).

En interrogeant avec Vygotskij les réalités épistémologiques et pratiques de l'éducation au $\mathrm{XXI}^{\mathrm{e}}$ siècle, il semble possible de reconnaitre à nouveaux frais les dangers réels produits par les impératifs logico-formels d'une gestion du social (Jonas, 1973/2013). En normalisant les comportements perçus comme des «moyennes » de conduite ou comme ce qui est «acceptable », autour de schèmes réducteurs et d'a priori culturels qui se présentent au sens commun comme des actes de bienveillance, on rencontre, dans les milieux scolaires et éducatifs, de nouvelles formes de violences institutionnelles (Auclair, 2018). C'est probablement une ruse de la Raison, mais il en revient encore et toujours aux adultes responsables et conscients de leurs rôles de questionner les apories qui instrumentalisent la vie sociale, le devenir humain et l'imaginaire enfantin

\section{Références}

Auclair, D. (2018). «Violences biopsychosociales et autoritarisme : la normalité de l'enfant et la normalisation des comportements dans les écoles du XXIe siècle ». Dans É. Dugas (dirs.), Les violences scolaires d'aujourd'hui en question. Regards croisés et altérité (pp. 15-25). L'Harmattan. Articles VIOLENCES BIOPSYCHOSOCIALES ET AUTORITARISME : LA NORMALITÉ DE L'ENFANT ET LA NORMALISATION DES COMPORTEMENTS DANS LES ÉCOLES DU XXIE SIÈCLE, David Auclair (editionsharmattan.fr)

Auclair, D. (2020). Moralité, autorité, normalité : fondements épistémologiques et politiques des transformations éducatives dans les sociétés occidentales depuis le milieu du XIX siècle, thèse de doctorat en sociologie, Université du Québec à Montréal. Archipel. UQAM | Bibliotheques | Depot institutionnel

Blais, M.-C, Gauchet, M. \& Ottavi, D. (2010). Conditions de l'éducation. Stock. Bronckart, J.-P. (1977). Théories du langage. Une introduction critique. Pierre Mardaga. Bronckart, J.-P. (1985). "Vygotskij, une œuvre en devenir ». Dans B. Schneuwly \& J.P. Bronckart. (dirs.), Vygotskij aujourd'hui (pp. 7-21). Delachaux \& Niestlé. 
Bronckart, J.-P. (2002), «La conscience comme "analyseur" des épistémologies de Vygotski et Piaget ». Dans Y. Clot. (dir.), Avec Vygotski (pp. 27-53). La Dispute.

Bruner, J. (2008). Culture et modes de pensée. L'esprit humain dans ses ouvres. Retz.

Bruner, J. (2015). Car la culture donne forme à l'esprit. Retz.

Clot, Y. (2002). «Introduction ». Dans Y. Clot. (dir.), Avec Vygotski (pp. 17-24). La Dispute.

Clot, Y. (2003). «Vygotskij, la conscience comme liaison». Dans L. S. Vygotskij, Conscience, inconscient, émotions (pp. 7-59). La Dispute.

Clot, Y. (2012) «Psychologie : une crise aggravée ?». Dans Y. Clot (dir.), Vygotskij maintenant (pp. 135-149). La Dispute.

Ducret, J.-J. (1984). Jean Piaget savant et philosophe, Tome I. Les années de formation, 19071924 \& Tome II. Étude sur la formation des connaissances et du sujet de la connaissance. Droz.

Dumont, F. (1981). «La culture savante : reconnaissance de terrain », dans Questions de culture, $\mathrm{n}^{\mathrm{o}}$ 1. IQRC. Fernand Dumont, La culture savante : reconnaissance du terrain. Questions de culture, Cette culture qu'on appelle savante. (uqac.ca)

Durkheim, É. (2014). Sociologie et philosophie. PUF.

Geertz, C. (2002). « Culture, esprit, cerveau ». Dans F. Rastier \& S. Bouquet (dirs.), Une introduction aux sciences de la culture (pp. 229-241). PUF.

Hegel, G. W. F. (1990). Textes pédagogiques. Vrin.

Jonas, H. (1973/2013). « Technologies et responsabilité. Réflexions sur les nouvelles tâches de l'éthique », dans Essais philosophiques. Du credo ancien à l'homme technologique, pp. 2546. Vrin.

Leopoldoff Martin, I. \& Schneuwly, B. (2012). « Le travail pédologique de Vygotskij : une réponse au diagnostic de la crise en psychologie?». Dans Y. Clot (dir.), Vygotskij maintenant (pp. 61-77). La Dispute.

Leopoldoff Martin, I. \& Schneuwly, B. (2018). «Introduction». Dans Vygotskij, La science du développement de l'enfant (pp. 7-46). Peter Lang.

Ottavi, D. (2009). De Darwin à Piaget. Pour une histoire de la psychologie de l'enfant. CNRS.

Piaget, J. (1997). L'éducation morale à l'école. De l'éducation du citoyen à l'éducation internationale. Anthropos.

Quentel, J.-C. (2008). L'enfant n'est pas une «personne ». Yapaka. https://hal.archivesouvertes.fr/hal-01983773/document

Schneuwly, B. (1997). "Psychologie et pédagogie : le paradigme psychopédagogique et son contraire », Cahiers de la recherche et du développement, no 7, pp. 27-44. Psychologie et pédagogie : le paradigme psychopédagogique et son contraire 1 Archive ouverte UNIGE

Sève, L. (1997). «Présentation ». In L. S. Vygotskij, Pensée et langage (pp. 11-32). La Dispute.

Sève, L. (2002). « Quelles contradictions ? à propos de Piaget, Vygotskij et Marx ». Dans Y. Clot (dir.), Avec Vygotskij (pp. 245-264). La Dispute.

Spencer, H. (1866). Education : intellectual, moral and physical. Appleton and company.

Vygotskij, L. S. (1985). «Textes de Vygotskij». Dans B. Schneuwly \& J.-P. Bronckart (dirs.), Vygotskij aujourd'hui (pp. 25-117). Delachaux \& Niestlé.

Vygotskij, L. S. (1983/1994). Défectologie et déficience mentale. Delachaux \& Niestlé.

Vygotskij, L. S. (1997). Pensée \& langage. La Dispute.

Vygotskij, L. S. (2018). La science du développement de l'enfant. Peter Lang. 